


\title{
FUNKTIONENTHEORETISCHE VORLESUNGEN.
}

\author{
VoN \\ DR. HEINRICH BURKHARDT, \\ o. PROFESSOR AN DER TECHNISCHEN HOCHSCHULE MÜNCHEN.
}

ERSTEN BANDES ERSTES HEFT.

A LGEBRAISCHE ANALYSIS.

LEIPZIG

VERLAG VON VEIT \& COMP.

1908 


\title{
ALGEBRAISCHE ANALYSIS.
}

\author{
VoN \\ Dr. HEINRICH BURKHARDT, \\ O. PROFESSOR AN DER TECHNISCHEN HOCHSCHULE MÜNCHEN.
}

ZWEITE, DURCHGESEHENE UND VERMEHRTE AUFLAGE.

MIT FIGUREN IM TEXT.

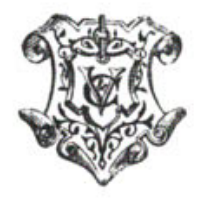

LEIPZIG

VERLAG VON VEIT \& COMP. 
